\title{
Modelling the Emergence and Evolution of e-Business Ecosystems from a Network Perspective
}

\author{
Ruize GAO ${ }^{1}$, Zhenji ZHANG ${ }^{1}$, Zhihong TIAN ${ }^{1}$, Xiaolan GUAN ${ }^{2}$, Ang $^{3}{ }^{3}$ \\ ${ }^{1}$ School of Economics and Management, Beijing Jiaotong University, \\ 3 Shang Yuan Cun, Haidian District, Beijing, BJ, 10, China, \\ 12113146@bjtu.edu.cn, zhjzhang@bjtu.edu.cn,10113130@bjtu.edu.cn \\ 2 Beijing Institute of Graphic Communication, \\ 1 Xinghua Avenue (Band Two), Daxing District, Beijing, BJ, 10, China, \\ 08113101@bjtu.edu.cn \\ 3 School of Business, Renmin University of China, \\ 59 Zhongguancun Street, Haidian District, Beijing, BJ, 10, China, \\ panghuai@126.com
}

\begin{abstract}
In recent years, we notice that the cooperation and competition among enterprises become much more complicated. Managers have to pay more attention to external cooperation from an ecological view. As e-business adoption becomes more pervasive, business ecosystems are shifting to e-business ecosystems. Because the situation is becoming more serious, in order to control the e-business ecosystem and earn profit from it, it is necessary for us to learn its structure and evolution. From the perspective of network science, this paper tries to connect complex network theories with e-business ecosystem research. We firstly analyse network structure of e-business ecosystem. Then an evolutionary model is proposed to describe the emergence and evolution of it. We finally use simulation and empirical methods to valid the theory we proposed.
\end{abstract}

Keywords: business ecosystem, e-business ecosystem, e-business, ecological evolution, complex network

\section{Introduction}

Decades ago, managers used to pay attention mostly on external competition. However, information and communications technology has dramatically changed business environment as well as the business models, especially for the last two decades. With the expanding market boundary and the increasingly fierce market competition, it became increasingly difficult for a single firm to succeed and thrive alone. This situation of firms becomes similar to that of individual plants and animals. Therefore, people begin to think deeply about the disadvantages of excessive competition and attach importance to external collaboration. It is proved that collaboration among supply chain partners could reduce inventory levels because of aggregation and that there is generally an overall benefit of collaboration. (Bhoon, \& Wadhwa, 2004) The profit levels of the whole supply chain and its companies are found to increase because of exchanging information through collaboration. (Jorge, Josefa, Raul, \& Juan, 2011) Also, inter-firm collaboration brings larger potential for knowledge creation especially for alliance networks "exhibit both high clustering and high reach". (Schilling, \& Phelps, 2007) There are positive effects that this kind of collaboration, especially which between users and producers or between competitors, has on company competitiveness and performance. (Álvarez, Marin, \& Fonfría, 2009) An example of this kind of collaboration is collaborative filtering recommendation for ebusiness e-retailers. (Jing, \& Liu, 2013)

In 1993, Moore put forward a new concept, business ecosystem. He presented the view that a company should be seen as a part of a business ecosystem instead of a member of an industry. And he indicated that, "in a business ecosystem, companies co-evolve capabilities around a new innovation: they work cooperatively and competitively to support new products, satisfy customer needs, and eventually incorporate the next round of innovations". (Moore, 1993) The concept of business ecosystem has a great influence on later studies. (Moore, 1996; Levien, 2004; Peltoniemi, \& Vuori, 2004; den Hartigh, Tol, \& Visscher, 2006; Anggraeni, Den Hartigh, \& Zegveld, 2007; etc) There are also several relative concepts, including industrial ecosystem, economy as an ecosystem, digital business ecosystem, etc. (Peltoniemi, \& Vuori, 2004).

For the past years, the Internet has had a profound effect on the economy. Firms began to adopt e-business to assist and improve their internal and external business processes. It implies "changes in the firm's strategy, 
management and marketing". (BordonabaJuste, Lucia-Palacios, \& Polo-Redondo, 2012) E-business adoption impacts performance by promoting both intra- and interorganizational collaboration. (Sanders, 2007)

"The overall intensity of e-business adoption" had a significant influence on measures of performance including efficiency, sales performance, customer satisfaction, and relationship development. (Wu, Mahajan, \& Balasubramanian, 2003) With the pervasive adoption of e-business, there was a notable shift from business ecosystem to e-business ecosystem. Nowadays, every firm has inevitably become a part of an e-business ecosystem and occupied a certain status in it. "The concept of e-commerce ecosystem is derived from business ecosystem" and "it is an inevitable form when business ecosystem develops to an advanced stage". (Liu, Tian, \& Guan, 2013) Therefore, it is time to concern on some new characteristics of ebusiness ecosystem.

We define an e-business ecosystem as an ecosystem in which, through Internet and with the support of ICT, all organizations (including suppliers, customers, logistics companies, financing institutions, certification authority, ecommerce platform providers, software companies, application providers, etc.) and individuals communicate and interact with each other to achieve co-evolvement. The technical environment of an e-business ecosystem weakens time and space boundaries. Enterprises have to enhance core competence and complementary advantages to survive. Conversely, organizations and individuals also influence the environment of ecosystem: they affect policy, regulations as well as social ideology and promote technical progress.

In e-business ecosystem, the ever-intense competition demands enterprises attaching much more importance on external collaboration. This kind of collaboration is to the benefit of mutual assistance, resources reassignment and the evolvement of the community. Competition is more common among communities. As the former president of PayPal, Scott Thompson once pointed out an interesting but universal thing about Internet to be that, in Internet age, two enterprises may cooperate for part of business while competing intensely. In this paper, we call competitioncooperation relationship co-opetition. (Brandenburger, \& Nalebuff, 1997) By now, there are few studies on structure and evolution of it from a global view. But obviously, this kind of study is of great significance in terms of promoting economic achievements and ecosystem evolvement. With the adoption of ebusiness, business ecosystem has become increasingly complicated. From a network perspective, it is notable that co-opetition in ebusiness ecosystem has formed into a network instead of simple bilateral relations. (Ritter, Wilkinson, \& Johnston, 2004; Anggraeni, Den Hartigh, \& Zegveld, 2007; Battini, Persona, \& Allesina, 2007; Braha, Stacey, \& Bar-Yam, 2011; etc) However, it has become difficult to accurately and efficiently describe the structure and dynamic changing of this kind of networks.

Meanwhile, complex network theory has been developed rapidly and had numerous theoretical achievements. (Watts, \& Strogatz, 1998; Barabási, Albert, \& Jeong, 2000; Bianconi, \& Barabási, 2001; Li, \& Chen, 2003; Barrat, Barthélemy, \& Vespignani, 2004; Costa, Rodrigues, Travieso, \& Villas Boas, 2007; Liu, Slotine, \& Barabási, 2011; etc) Based on complex network theory, real social networks can be quantitatively described and analysed by methods and tools of statistical physics. It has been widely applied in several research fields. Several studies have showed that business network display some general features of complex networks. (Braha, Stacey, \& Bar-Yam, 2011; Chen, \& Lin, 2012; etc) For example, supply chain network is "with the characteristics of small-world network and scale-free network". (Chen, \& Lin, 2012) Therefore, it might be a practical tool for studying e-business ecosystem from a global view. Important theories of complex networks, like evolution, dynamics of spreading and synchronization, would have much practical significance in this study. By making use of them, it brings convenience to analyse the emergence and evolution of e-business ecosystem precisely. And it may bring new insights in improving the business system.

In section 2, we propose a conceptual network for an e-business ecosystem by setting nodes and edges. In Section 3, the structural characteristics of the network are analysed. In section 4, in consideration of real features, this paper develops a dynamic network model to describe the emergence and evolvement of e-business ecosystems. Following that, there is the simulation work. In section 5, by taking SMEs on the third 
party e-commerce platform Alibaba.com as an example, we come to some practical implications through an empirical research.

\section{Networking}

\subsection{Nodes}

As subjects in an e-business ecosystem, enterprises in it can be regarded as nodes of a network. It is obvious that there could be different kinds of enterprises including suppliers, consumers, agencies, logistics companies, consulting companies, online marketers, financing institutions, third-party payment platform, certification authority, ecommerce platform providers, Internet access providers, software companies, application service providers, etc. (Figure 1.)

In an e-business ecosystem, different enterprises play different roles and display different capability. For example, as one of the biggest e-commerce platform, Alibaba.com plays the role of resources integrator and coordinator. Logistics companies, like UPS, provide only logistics services. And various SMEs on Alibaba.com can be suppliers and consumers and enjoy services provided by the platform and others.
Thus parties involved in an e-business ecosystem can be regarded as nodes of a network. Weight and attribute could be assigned to each node in order to distinguish them.

\subsection{Edges}

In natural ecosystems, different organisms usually compete and at the same time cooperate with each other. It is the same as in e-business ecosystems. Actually the reason for the concept of business ecosystem being raised is precisely because people have begun to think about the disadvantage of excessive competition and the necessary of cooperation. In electronic age, this kind of co-opetition has become more complicated, more generalized and closer. In an e-business ecosystem, two enterprises could cooperate for part of business while competing intensely.

Co-opetition in an e-business ecosystem can be regarded as edges of a network. Due to their different patterns, those edges display diverse attributes. When the relationship is unilaterally beneficial, the edge is oriented. But more often it is bidirectional. The scale of co-opetition could be displayed by assigning weight. Generally, a larger weight means a closer relationship and quicker information transfer.

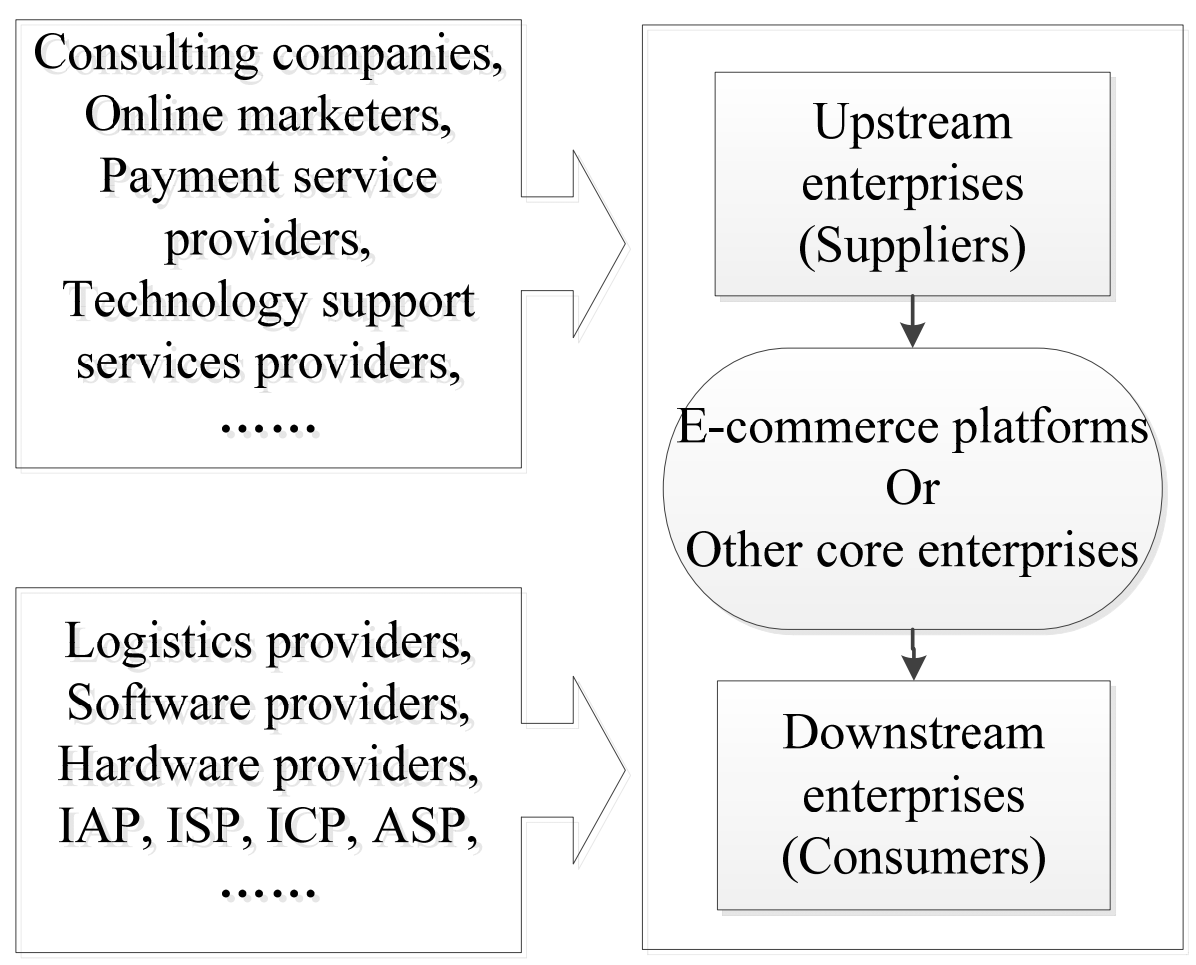

Figure 1. Enterprises in e-business ecosystem 


\section{Characteristics Analysis of the Network}

\subsection{Community}

In electronic ages, cooperation becomes much more important. The increasingly complicated and rapidly changing market demands a lot of attention not only on improving internal capabilities but also on external cooperation. External cooperation is good for the complementation of core competitiveness, resources integration and optimizing the enterprise community as well as the whole ecosystem. A community emerges when several enterprises jointly launch close coopetition. When emphasizing cooperation inside a community, competition exists among different communities instead of different enterprises. It means that the relationships inside the community are pretty close and beneficial for each, while relatively sparse relationships exist among different communities. Through analysis on community structure, local features of a network can be revealed. It is useful for decision optimization of enterprises.

\subsection{Hierarchy}

The network has hierarchy. Upstream enterprises and downstream enterprises form different layers of the network (as we discussed above). Relationship among them is vertical. Those enterprises cooperate when creating value. And they compete when sharing value. Horizontal relationships exist between enterprises which compete with each other with some complementary advantages. In e-business ecosystem, they may share purchasing platform and sales platform to share information, so as to reach agglomerate effect, to increase bargaining power and to explore more chances. Therefore, the network can be multilevel and both vertical and horizontal relationships appear to be co-opetition.

\subsection{Dynamic evolution}

A business ecosystem as a whole is greater than the sum of its parts. (Peltoniemi, \& Vuori, 2004) It is the same for the situation of electronic age, i.e. e-business ecosystem. It is notable that ebusiness ecosystem is an open system, in which the number of enterprises constantly changes: new nodes enter and the old ones exit. An example is that a number of traditional retailing enterprises implementing e-commerce but failed. Besides, the uncertainty of establishing or terminating relationships can also be reflected on different behaviour incentive of nodes (enterprises). Generally, enterprises launch cooperative relationships following some preferential mechanism, which will be discussed in detail in next section.

\section{Modelling \& simulation}

\subsection{The basis}

BA model (Barabási, Albert, \& Jeong, 2000) simplifies the scale-free characteristic to two evolutionary mechanisms: growth and preferential attachment. In BA model, the preferential probability is (Barabási, Albert, \& Jeong, 2000)

$$
\Pi_{i}\left(k_{i}\right)=k_{i} / \sum_{i} k_{i} .
$$

It is a linear function of degree $\mathrm{k}$. This mechanism leads to a phenomenon called rich club. On one hand, considering the real situation of e-business ecosystem, the number of an enterprise's co-opetition (degree) reflexes its internal capability and therefore its attraction to others, which leads to a rich club. On the other hand, it is notable that the attraction of an enterprise also relates to some internal properties of it, called fitness (fitness model, Bianconi, \& Barabási, 2001). That is, a newly coming enterprise with a larger fitness is also more attractive. Thus, as the core and key-nodes of the network, hub enterprises are not necessarily those old ones. Usually, core enterprises in an e-business ecosystem have stronger comprehensive capability in capital, technology, products, services, etc. and occupy huge market shares. They are leaders of their communities. (Levien, 2004) More than that, all the hub-firms collectively play leading roles in the entire e-business ecosystem. They are of great research significance because they lead to the robust yet fragile properties of the network. Other firms with weak capabilities have limited resources and external relationships. Moreover, when choosing a cooperation partner, especially for a SME, global information is usually inaccessible even if with the most advanced technology. Therefore preferential attachment may be 
limited to some local area, which leads to a local world model. (Li, \& Chen, 2003) What's more, if considering scale of coopetition as the factor influencing preferential attachment, a weighted evolutionary model can be founded (e.g. BBV model, Barrat, Barthélemy, \& Vespignani, 2004).

Above all, the emergence and evolvement process of an e-business ecosystem is of much complexity. Considering those real features, we are going to propose a reasonable improved model for specific requirements of research.

\subsection{Attachment mechanism}

As discussed in section 2, different edges and nodes in an e-business ecosystem have different weights. We assume that, between nodes $i$ and $j$, there might be several cooperative projects and each will contribute to the corresponding edge weight. Let $w_{i j}$ be the weight of the edge between $i$ and $j$. Let $s_{i}$ be the weight of node $i$,

$S_{i}=\sum_{j} w_{i j}$.

In an e-business ecosystem, a dynamic fitness $\beta(\in[0,1])$ can be assigned to each enterprise to indicate intrinsic ability of innovation, specialty technology, competitiveness and other advantages. Fitness $\beta$ is chosen from a distribution $P(\beta)$, which is normally a Gaussian distribution. Fitness $\beta$ can constantly change, let $\beta_{i}=\beta_{i}(t)$. If intrinsic ability of an enterprise is greater, it can establish more co-opetition relationships. It means that the number of edges a new node $i$ builds with existing nodes, $m_{i}$, is affected by fitness, let $m_{i}=f\left(\beta_{i}\right)$. Also, the scale of newly established relationship between $i$ and $j$ is affected by $\beta_{i}, \beta_{j}, \quad s_{i}$ and $s_{j}$, let $\Delta w_{i j}=P\left(\beta_{i}, s_{i}, \beta_{j}, s_{j}\right)$.

What's more, existed co-opetition relationships (degree) of a firm can be some preferable quality attracting new relationships. This attraction is better represented by node weight, which consists of both node degree and edge weight. But a firm with greater fitness could also be chosen with a larger probability. Therefore, the probability of node $i$ being chose to connect with a new node relates to both weight $s_{i}$ and fitness $\beta_{i}$. The preferential probability is
$\Pi_{i}\left(s_{i}, \beta_{i}\right)=\frac{\beta_{i} s_{i}}{\sum_{j} \beta_{j} s_{j}}$.

$\beta_{i} s_{i}$ is the integrated ability of enterprise $i$, let it be $D_{i}$.

Furthermore, in an e-business ecosystem it is unlikely for an enterprise to have the global information of the network. Inspired by the local-world model ( $\mathrm{Li}$, \& Chen, 2003), it is more practical if we assume that an enterprise establishes co-opetition relationships with others through local information. The initial local world of a new enterprise is determined by randomly choosing $M$ existing enterprises. Let the size of a local world $M_{i}=g\left(\beta_{i}\right)$. And it can be assumed that after enterprise $i$ established co-opetition relationship with $j$, the local world of $j$ becomes an additional part of $i$ 's. In this way, the effect of local-world mechanism would vanish as the evolution goes on. Besides, a local world should not be too small, otherwise the preferential attachment mechanism cannot function well. Considering local information, the preferential probability of node $i$ to be connected with $j$ is

$$
\begin{aligned}
& \Pi_{i}\left(s_{i}, \beta_{i}\right)=P\left(i \in \text { local }_{j}\right) * \frac{D_{i}}{\sum_{s \in l o c a l_{j}} D_{s}} . \\
& P\left(i \in \text { local }_{j}\right)=M_{j} / \mathrm{N}(\mathrm{t})
\end{aligned}
$$

\subsection{Growing mechanism}

Like organisms in a natural ecosystem, enterprises in an e-business ecosystem have their life cycles. The growing of an e-business ecosystem is nonlinear and periodic. Its evolution can be roughly divided into 2 periods: emerging and rapidly growing period $N_{1}$ and mature period $N_{2}$. The evolution process is:

1. The initial network consisted of $n_{0}$ nodes, which are linked to each other.

2. In emerging and rapid growth period, the enthusiasm of cooperating and competing is great and a lot of new firms will enter. A new enterprise $i$ builds co-opetition relationships with $m_{i}$ existing enterprises. These $m_{i}$ enterprises should be chosen through the preference mechanism as above.

3. In mature period, the growing is almost stopped. There are four possible situations: 
(1) a new enterprise may enter; (2) old coopetition may be removed because the cooperation becomes due or other reasons; (3) new co-opetition relationships are established; (4) some existed enterprises may exit the network. Therefore, at every time $t$ in this period, the network involves through one of those situations with certain probabilities:

- Add nodes with probability $p$ : The same as in rapid growth period;

- Delete nodes with probability $q(\approx p)$ : Randomly select an enterprise from the network. If its integrated ability $D_{i}$ is lower than a certain proportion $a$ (e.g. $a=1 / 2$ ) of average ability, delete it and all its co-opetition relationships (edges);

- Add edges with probability $r\left(\approx 0.5^{*}(1-\mathrm{p}-\mathrm{q})\right): \quad$ Randomly select firms $i$ and $j$ and establish a new cooperative relationship with weight $\Delta w_{i j}$ between them;

- Delete edges with probability 1-p-q-r: Randomly select adjacent firms $i$ and $j$ and broke the link between them. Firm $i$ is chosen by reversepreference probability

$\Pi_{i}{ }^{\prime}=\left(1-\Pi_{i}\right) /(N(t)-1)$.

Surely this model can be further improved or adjusted to match the requirement of research, for example, by adding more complicated stages, by changing the initial state, by altering the rule of adding notes and establishing new edges. Also more restriction can be added, for example, hub-nodes mustn't be removed. Furthermore, the network could be set as a multilevel network.

\subsection{Simulation}

Through MATLAB the model can be simulated. At the end of the evolution process $(t=8000)$, the distribution of node weight follows a power-law distribution to some extent (Figure 2.).

As the evolution goes on, the power low fitting index of node weight at different time point is display in Figure 3.

It is clear that, at the very beginning of the first period, the power law fitting index fluctuates a lot. And then it increases relatively smoothly. In the second period, it remains steady around 2.37.

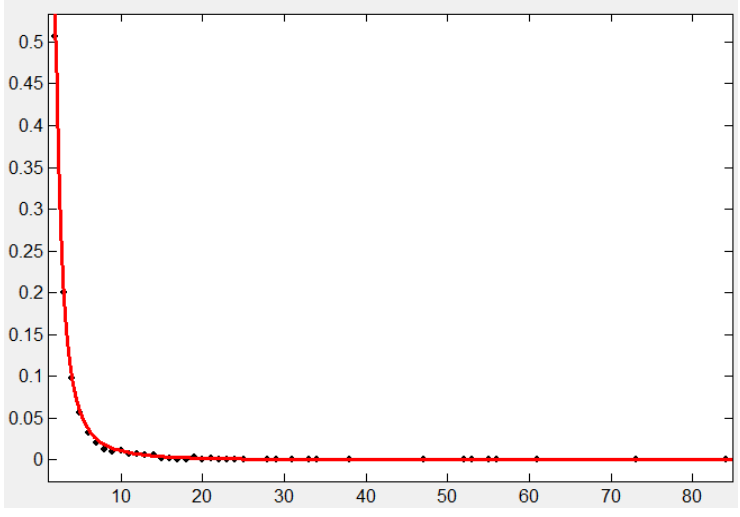

Figure 2. Power low fitting of node weight at $\mathrm{t}=8000$ : power law index $\gamma=2.4, \mathrm{R}^{2}=0.9995$, $\mathrm{SSE}=0.0001467$ (horizontal axis: node weight; vertical axis: probability)

Parameter setting: $\beta_{i} \sim N(0.5,0.01), n_{0}=3$, $m_{i}=f\left(\beta_{i}\right)=2, \Delta w_{i j}=P\left(\beta_{i}, s_{i}, \beta_{j}, s_{j}\right)=1, N_{l}=6000$, $N_{2}=8000, p=0.4, q=0.4, r=0.2, M_{i}=N(t)$.

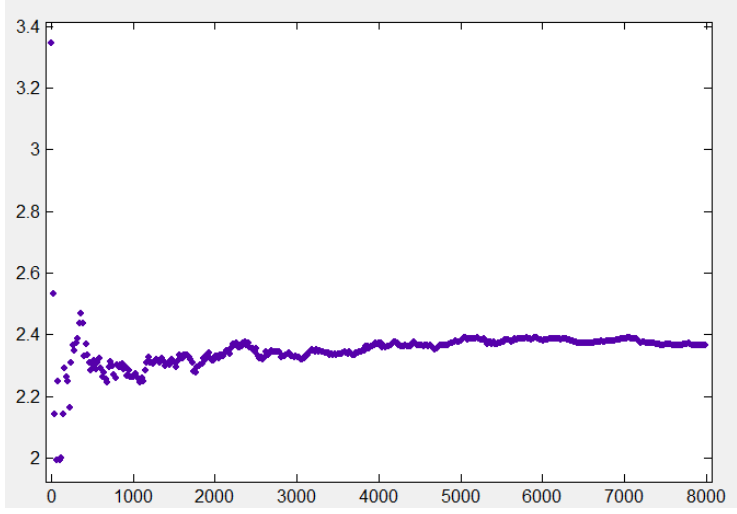

Figure 3. Power low fitting index of node weight as evolution goes on (horizontal axis: time; vertical axis: power law index)

Simulation shows that the node degree has the similar characteristics as node weight above. And by UCINET, the clustering coefficient is $C$ $=0.031$. The average path length is $L=3.021$. This means the network is small-world.

\section{Empirical analysis \& discussion}

\subsection{Empirical analysis}

In America, usually a few large enterprises coexist in an industry. They have constructed ebusiness/e-commerce system of their own and become the leading force of e-business development of the industry, for example, Covisint, the joint procurement platform for General Motors, Chrysler and Ford. Other examples are like the e-business system of Intel 
and Dell. In China, there are few enterprises having their own e-business platform. The vast majority, most of which are SMEs, use the third party e-commerce platform. By this, SMEs can save a lot of money and labour force on the implementation of e-business. And it is conducive to resource integration. Therefore, in consideration of the practical situation of China and data availability, we will analysis the SMEs on Alibaba.com, which is the largest third party e-commerce platform in China.

This paper takes textile machine manufacturers as an example. Information about 182 of them on Alibaba.com is collected, as well as number of co-opetition of each (node degree).

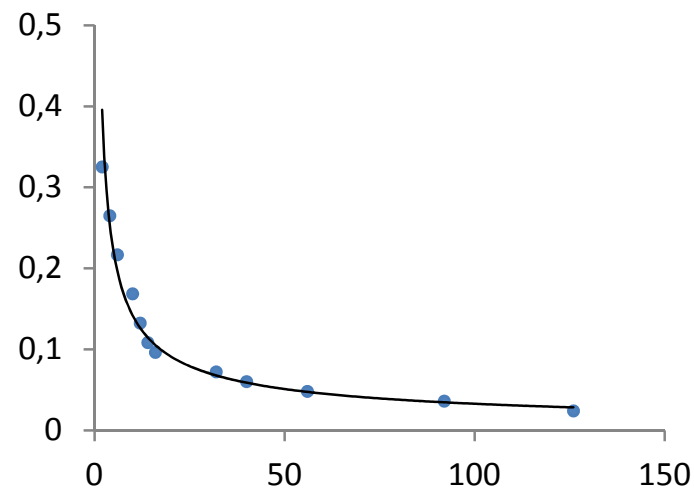

Figure 4. Power low fitting of node degree of textile machine manufacturers on Alibaba.com: $\gamma=2.137$, $\mathrm{R}^{2}=0.9827$ (horizontal axis: node degree; vertical axis: probability)

It is clearly that node degree distribution of textile machine manufacturers on Alibaba.com is approximately power-law. From the original data, it is found that the largest node degree is 124. Node degrees of most enterprises are far less than this number. It shows that there are a few core enterprises in them.

\subsection{Discussion}

\subsubsection{Core enterprises}

In social network analysis, closeness (Freeman, 1979) of a node measures how close it is to all other nodes. Considering weights, this paper defines closeness of $i$ as

$C_{\mathrm{c}}^{\prime}(i)=\frac{N-1}{\sum_{j=1}^{N} D_{i j}}$,

$D_{i j}=1 / w_{i k}+1 / w_{k l}+\ldots+1 / w_{n},\{i, k, l, \ldots, n, j\}$ is the shortest path between $i$ and $j$. However, closeness is defined only if the network is strongly connected, otherwise there might be no path between $i$ and $j$. Enterprises with larger closeness are affected more rapidly by information and technology transferring. And conversely, they can exert greater influence on the whole network.

In an e-business ecosystem, positive relationships may create synergistic effect. Otherwise, the prospective benefits will be difficult to achieve and enterprises would take losses. From last section, it is known that with increasing complicated relationships among enterprises and with the evolvement of the ebusiness ecosystem, the distribution of node degree is increasingly asymmetric and shows a power-law trend and core enterprises emerge. Actually there is a prerequisite for the emergence of core enterprises. That is the asymmetrical distribution of information, resources and capability in the ecosystem. Those enterprises, which become the core, not only possess the core technology and resources, but also have greater synthetic ability especially risk resisting capability. Coordination and control of e-business ecological communities might be more effective by a few core enterprises than forced control from hierarchical management. Therefore, the emergence of core enterprises is beneficial for evolvement of the whole ebusiness ecosystem.

\subsubsection{Efficiency}

Functions of e-business ecosystem include resources integration and value creation and sharing. The global efficiency of information spreading in it can be defined as below

$$
E=\frac{1}{N(N-1)} \sum_{i \neq j} w_{i j},
$$

When $E$ is close to 1, the network is globally efficient. Besides, average path length $L$ (Watts, \& Strogatz, 1998) measures the separation degree as well as the efficiency of information flows, because a long path usually results in resources loss. By contrast, local efficiency can be described by clustering coefficient $C$ (Watts, \& Strogatz, 1998), which reflects link density, small-world property, group phenomenon, connectivity and transitivity. Network efficiency can be enlarged through breaking and rewiring edges (terminating and establishing co-opetition relationships). 
According to small-world theory, establishing a few long-range paths between the principal nodes is positive to decrease path length so as to promote the global efficiency of the network. However, complex network theory also indicates that it is far from positive if there are a big number of random shortcuts of this kind: it may arouse disorder of technology and information transmission and cause chaos. For an e-business ecosystem, a larger clustering degree means faster information spreading and a more efficient network, while an overlarge clustering degree would lead to homogenization and bring difficulty in achieving balance of it. Thus, positive control of the network efficiency requires sophisticated study on those parameters and observation on both local and global structure of e-business ecosystem.

\subsubsection{Robustness}

The vulnerability of an enterprise $i$ is $V_{i}=(E$ $\left.E_{i}\right) / E$. (Costa, Rodrigues, Travieso, \& Villas Boas, 2007) $E$ is global efficiency. $E_{i}$ is global efficiency after extracting node $i$ from the network. The network vulnerability is $V=\max$ $\left\{V_{i}\right\}$. Through this, the most fragile enterprises could be identified. Protecting and supporting those firms could be an effective way to protect the community as well as the whole ecosystem.

Usually the core enterprises occupy huge numbers of links in a community. The rest of links and enterprises make the network structure more complex and stable. It adds to the robustness of the system. If random attack occurs on the e-business ecosystem, it is less likely to happen on core enterprises and will not have much effect on structure and function of the community or ecosystem. But once problems occur on core enterprises, the influence would spread quickly throughout the community and even lead to collapse of it. Therefore, core enterprises are worth high attention. To avoid excessive dependence on core enterprises, some particular co-opetition relationships should be encouraged.

\section{Conclusions}

For years, people have gradually realized that e-business ecosystems are forming and that co-opetition among e-business enterprises is worthy of researches. This paper focuses on the structure and evolution process of e- business ecosystems, which is of great use to decision making for this kind of enterprises and administration. We construct a conceptual network for e-business ecosystem and analyze the community and hierarchy structure of it as well as its dynamic characteristic. And then based on BA model, a staged weighted model is proposed, in which fitness and local-world mechanisms are comprised and the evolution process is divided into two periods. Through simulation, it is found that the distribution of both node weight and node degree follows a power-law distribution to some extent. Following that, by an empirical study on the SMEs on Alibaba.com, some points are drawn. Ebusiness ecosystem is a new concept in business research. Further research can be carried out as empirical study. Analysis on some specific cases through this model is of certain practical significance to regulation and control.

\section{REFERENCES}

1. ÁlVAREZ, I., R. MARIN, A. FONFRÍA, The Role of Networking in the Competitiveness of Firms, Technological Forecasting and Social Change, vol. 73, no. 3, 2009, pp. 410-421.

2. ANGGRAENI, E., E. DEN HARTIGH, M. ZEGVELD, Business Ecosystem as a Perspective for Studying the Relations between Firms and Their Business Networks, In ECCON 2007 Annual meeting, 2007, October.

3. BARABÁSI, A. L., R. ALBERT, H. JEONG, Scale-free Characteristics of Random Networks: the Topology of the World-Wide Web, Physica A: Statistical Mechanics and its Applications, vol. 281, no. 1, 2000, pp. 69-77.

4. BARRAT, A., M. BARTHÉLEMY, A. VESPIGNANI, Modeling the Evolution of Weighted Networks, Physical Review E, vol. 70, no. 6, 2004, pp. 066149.

5. BATTINI, D., A. PERSONA, S. ALLESINA, Towards a Use of Network Analysis: Quantifying the Complexity of Supply Chain Networks, International Journal of Electronic Customer Relationship Management, vol. 1, no. 1, 2007, pp. 75-90. 
6. BHOON, K. S., S. WADHWA, Challenges and Opportunities in Information Enabled Supply Chain Collaboration, Studies in Informatics and Control, vol. 13, no. 4, 2004, pp. 285-294.

7. BIANCONI, G., A. L. BARABÁSI, Competition and Multiscaling in Evolving Networks, EPL (Euro Physics Letters), 2001, vol. 54, no. 4, p. 436.

8. BORDONABA-JUSTE, V., L. LUCIAPALACIO, Y. POLO-RRDONDO, Antecedents and Consequences of eBusiness Adoption for European Retailers, Internet Research, vol. 22, no. 5, 2012, pp. 532-550.

9. BRAHA, D., B. STACEY, Y. BAR-YAM, Corporate Competition: A Selforganized Network, Social Networks, vol. 33, no. 3, 2011, pp. 219-230.

10. BRANDENBURGER, A. M., B. J. NALEBUFF, Co-Opetition: A Revolution Mindset That Combines Competition And Cooperation: The Game Theory Strategy That's Changing, Currency Doubleday, 1997.

11. CHEN, H. H., A. M. LIN, Complex Network Characteristics and Invulnerability Simulating Analysis of Supply Chain, Journal of Networks, vol. 7, no. 3, 2012, pp. 591-597.

12. COSTA, L. D. F., F. A. RODRIGUES, G. TRAVIESO, P. R. VILLAS BOAS, Characterization of Complex Networks: A Survey of Measurements, Advances in Physics, vol. 56, no. 1, 2007, pp. 167-242.

13. den HARTIGH, E., M. TOL, , W. VISSCHER, The Health Measurement of a Business Ecosystem, Proceedings of the European Network on Chaos and Complexity Research and Management Practice Meeting, 2006, October.

14. FREEMAN, L. C., Centrality in Social Networks Conceptual Clarification, Social Networks, vol. 1, no. 3, 1979, pp. 215-239.

15. JING, Y., H. LIU, A Model for Collaborative Filtering Recommendation in E-Commerce Environment, International Journal of Computers Communications \& Control, vol. 8, no. 4, 2013, pp. 560-570.
16. JORGE, E. H., M. JOSEFA, P. RAUL, P. JUAN, A Multiagent Negotiation Based Model to Support the Collaborative Supply Chain Planning Process, Studies in Informatics and Control, vol. 20 , no. 1, 2011, pp. 43-54.

17. LEVIEN R., The Keystone Advantage: What the New Dynamics of Business Ecosystems Mean for Strategy, Innovation, and Sustainability, Harvard Business School Press, 2004.

18. LI, X., G. CHEN, A Local-world Evolving Network Model, Physica A: Statistical Mechanics and its Applications, vol. 328, no. 1, 2003, pp. 274-286.

19. LIU, H. L., Z. H. TIAN, X. L. GUAN, A New Conceptual and Evolution Model for E-commerce Ecosystem, AISS: Advances in Information Sciences and Service Sciences, vol. 5, no. 4, 2013, pp. 630-638.

20. LIU, Y. Y., J. J. SLOTINE, A. L. BARABÁSI, Controllability of Complex Networks, Nature, vol. 473, no. 7346, 2011, pp. 167-173.

21. MOORE, J. F., Predators and Prey: a New Ecology of Competition, Harvard Business Review, vol. 71, no. 3, 1993, pp. 75-75.

22. MOORE, J. F., The Death of Competition: Leadership and Strategy in the Age of Business Ecosystems, New York: Harper Business, 1996.

23. PELTONIEMI, M., E. VUORI, Business Ecosystem as the New Approach to Complex Adaptive Business Environments, Proceedings of E-business Research Forum, 2004, pp. 267-281.

24. RITTER, T., I. F. WILKINSON, W. J. JOHNSTON, Managing in Complex Business Networks, Industrial Marketing Management, vol. 33, no. 3, 2004, pp. $175-183$.

25. SANDERS, N. R., An Empirical Study of the Impact of E-business Technologies on Organizational Collaboration and Performance, Journal of Operations Management, vol. 25, no. 6, 2007, pp. 1332-1347. 
26. SCHILliNG, M. A., C. C. PHELPS, Interfirm Collaboration Networks: The Impact of Large-Scale Network Structure on Firm Innovation, Management Science, 2007, vol. 53, no. 7, pp. 1113-1126.

27. WATTS, D. J., S. H. STROGATZ, Collective Dynamics of 'Small-world'
Networks, Nature, vol. 393, no. 6684, 1998, pp. 440-442.

28. WU, F., MAHAJAN, V., S. BALASUBRAMANIAN, An Analysis of E-business Adoption and Its Impact on Business Performance, Journal of the Academy of Marketing Science, vol. 31, no. 4, 2003, pp. 425-447. 